\title{
AUC All Normalized by Body Mass Index
}

National Cancer Institute

\section{Source}

National Cancer Institute. AUC All Normalized by Body Mass Index. NCI Thesaurus. Code C92362.

The area under the curve (AUC) from the time of dosing to the time of the last observation divided by the body mass index, regardless of whether the last concentration is measurable or not. 